\title{
PHOTOOXIDATIVE CLEAVAGE OF OXAZOLINE-2-THIONE IN PROTIC AND APROTIC SOLVENTS
}

\author{
Indra Sen Singh \\ Chemical Engineering Department, School of Technology, Copperbelt University, \\ P.O. Box 21692, Kitwe, Zambia
}

(Received September 7, 1998; revised July 21, 1999)

\begin{abstract}
The photolysis of 4,5-diphenyl-3-(4-methylphenyl)-4-oxazoline-2-thione (a) in benzene or cyclohexane in the presence of singlet oxygen using Pyrex filtered $450 \mathrm{~W}$ Hanovia lamp afforded benzil (f), N-4-methylphenyl benzamide (h) and N,N-dibenzoyl-4-methylaniline (e). When the same reaction was performed in protic solvents, the formation of benzil ( $(\mathbf{4}, \mathrm{N}-4$ methylphenyl benzamide (h), N,N-dibenzoyl-4-methylaniline (e) and benzoic acid ( $g$ ) was observed. The products were characterized by spectral data and comparison (undepressed melting points and identical IR spectra) with authentic samples. Oxazolines are widely used class of compounds and therefore it would be relevant to study the effect of UV light on such compounds in different conditions. The present study derives an interesting route for the photooxidative fragmentation of 4-oxazoline-2-thione ring system. It involves a dioxetane derivative (b) which after cleavage gives two radicals $c$ and $d$ which finally give the above products. The yield of $\mathrm{N}-4$-methylphenyl benzamide is increased by protic solvents which gives a strong support to the proposed mechanism.
\end{abstract}

\section{INTRODUCTION}

The oxazoline ring system has assumed marked significance due to their wide application [1]. The synthetic utility of 2-oxazolines has been reviewed for aromatic systems in which they go for direct $o$-lithiation of benzene molecule [2]. The reactions of dianions from ketomonoimines with ethyl chlorofomate and carbon disulphide give substituted 4-oxazolin2-ones and 4-oxazoline-2-thiones [3]. 4-Oxazolin-2-ones have been converted to the corresponding -2-thiones by reaction with phosphorus pentasulphide [4]. The thermal rearrangement of isoxazoline-3-one to -4-oxazoline-2-one has been observed [5]. Photosensitised oxidation of 3,4,5-triphenyl-4-oxazoline-2-one gives a mixture of benzoic acid, benzanilide and ortho- and para-benzamide benzophenone [6]. A similar photodecarbonylation of a cyclic carbonate to 1,2-diketone derivative has been reported [7]. This study uses singlet oxygen and aims to explore the effects of protic and aprotic solvents. To provide further insight in the mechanistic details of photooxidative fragmentation of substituted 4-oxazoline-2-thione ring systems, different protic and aprotic solvent combinations were used. In this study photooxidative cleavage of substituted -4-oxazoline-2thione ring system in protic and aprotic solvents has been discussed. The reaction route explored in this study demonstrates an interesting chemistry.

\section{RESULTS AND DISCUSSION}

The starting material (a) was prepared using previously reported method [3]. Irradiation of a dilute solution of (a) in benzene in presence of oxygen with UV light ( $450 \mathrm{~W}$, Hanovia Lamp) 
afforded benzil (f), N-4-methylphenyl benzamide (h) and N,N-dibenzoyl-4-methylaniline (e). Benzil (f) was characterized by comparison (undepressed mixed melting point and superimposable identical IR spectrum) with authentic sample which was procured from Ega Chem. Germany. N-4-methylphenyl benzamide (h) was also characterized by spectral data and comparison (undepressed mixed melting points) with authentic samples. The authentic sample of N-4-methylphenyl benzamide was prepared from the reaction of benzoyl chloride and $p$-toluidine in the presence of potassium hydroxide [8]. $\mathrm{N}, \mathrm{N}$-dibenzoyl-4-methylaniline has been characterized on the basis of the spectral data. The reaction route for the formation of products by photooxidative fragmentation of -4-oxazoline-2-thione (a) can be depicted in Scheme 1.<smiles>CCN1SOC(c2ccccc2)C1c1ccccc1</smiles>

$\mathbf{a}$<smiles>CCCN1OSC2(c3ccccc3)OOC1(c1ccccc1)C2C(=O)[O-]</smiles><smiles>CCCN(C(=O)c1ccccc1)C(=O)c1ccccc1</smiles>

e

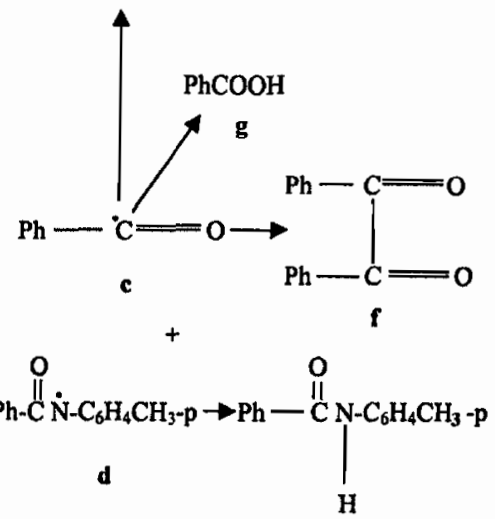

h

\section{Scheme 1}

The addition of singlet oxygen to -4-oxazoline-2-thione (a) gives the dioxetane derivative (b) which after fragmentation accompanied by loss of carbonyl sulfide could give rise to benzoyl radical (c) and $\mathrm{N}$-4-methylphenyl benzamide radical (d). The benzoyl radical (c) dimerises to yield benzil (f) and the amide radical (d) abstracts hydrogen to give N-4methylphenyl benzamide (h). The radical (c) and (d) combine to result in N,N-dibenzoyl-4methylaniline. Irradiation of a dilute solution of (a) in protic solvents in the presence of singlet oxygen with UV light (450 W Hanovia lamp) gave four products which were identified as benzil (f), benzoic acid (g), N-4-methylphenyl benzamide (h) and $\mathrm{N}, \mathrm{N}$ dibenzoyl-4-methylaniline (e). The products benzil (f) and benzoic acid (g), were characterized by comparison (undepressed mixed melting point and identical IR spectra) with 
authentic samples. As reported earlier, the products (e) and (h) have been characterized on the basis of their spectral data. The reaction route for the formation of products by the fragmentation of (a) in protic solvents seems to be similar to Scheme 1. The additional product, benzoic acid may arise from the benzoyl radical (d). The oxygenation to similar systems gave a mixture of benzoic acid, benzamide and ortho- and para-benzamide benzophenone [6]. It has been observed that reaction route involves a dioxetane derivative which is proposed on the basis of $\mathbb{R}$ spectrum of photolysis mixture before the completion of reaction. The presence of a weak band at $880 \mathrm{~cm}^{-1}$ gives a strong support for the presence of dioxetane. This derivative, however, could not be isolated due to its poor stability.

Irradiation of a dilute solution of $(a)$ in benzene using methylene blue as sensitzer in sun light gave three products which were identified as benzil (I), N-4-methylphenyl benzamide (h) and N,N-dibenzoyl-4-methylaniline (e) on the basis of comparison (undepressed mixed melting point and superimposable $\mathbb{R}$ spectrum) with the authentic samples. The similar irradiation in protic solvents afforded an additional product which was identified as benzoic acid (g) on the basis of comparison (undepressed mixed melting point and superimposable IR spectrum) with the authentic sample. The reaction route for the formation of above products is the same as in Scheme 1. These results offer a strong support for the proposed photooxidative cleavage.

The irradiation of a dilute solution of (a) in protic and aprotic solvents with $450 \mathrm{~W}$ Hanovia lamp in nitrogen atmosphere gave one product which was identified as benzilmono4-methylphenylimine on the basis of comparison (undepressed mixed melting point and superimposable IR spectrum) with authentic samples. A similar photodecarbonylation of a cyclic carbonate to 1,2-diketone derivative has been reported [7]. It has also been observed in the present study that the yield of $\mathrm{N}-4$-methylphenyl benzamide increases in protic solvents as compared to that in aprotic solvents which offers a strong support for the proposed mechanism of photooxidation in Scheme 1.

\section{EXPERIMENTAL}

Instrumentation. Chemicals and reagents were purchased from Aldrich Chemical Co. and used without further purification. Melting points were determined in capillaries on melting point apparatus and are uncorrected. The NMR spectra in $\mathrm{CDCl}_{3}$ was recorded on Varian A60D and VN 1009 (S-60T) spectrophotometer with TMS as an internal standard. The IR spectra were measured on a Perkin Elmer 720 spectrophotometer and UV spectra on a Beckman DB-G spectrophotometer. Photochemical experiments were carried out using Hanovia type A, $450 \mathrm{~W}$ medium pressure mercury immersion lamp as light source.

Preparation of 2-imino-1,2-diphenylethanone. The starting 2-imino-1,2-diphenylethanone was obtained by heating an equimolar mixture of benzil and suitable aromatic amine at $150^{\circ} \mathrm{C}$ for $2-3 \mathrm{~h}$ following the reported method and purified by fractional crystallization from ethanol (75-78\%) [9].

Preparation of 4,5-diphenyl-3-(4-methylphenyl)-4-oxazoline-2-thione (a). It was prepared by the reaction of dianions from a suitable 2-imono-1,2-diphenylethanone with carbon disulfide and purified by using a reported method [3]. The UV spectrum shows absorption maxima at $310 \mathrm{~nm}\left(\epsilon 1.60 \times 10^{4}\right)$ and $225 \mathrm{~nm}\left(\epsilon 2.25 \times 10^{4}\right)$. The IR spectrum exhibits a medium intensity band at $1605 \mathrm{~cm}^{-1}(\mathrm{VC}=\mathrm{C})$ and a strong band at $1162 \mathrm{~cm}^{-1}(\mathrm{VC}=\mathrm{S})$ [4]. The NMR 
spectrum displays the aromatic protons as a multiplet at $\delta 7.30(14 \mathrm{H})$ and methyl proton as a singlet at $\delta 2.30(3 \mathrm{H})$.

General procedure for irradiation. A dilute solution containing $0.5 \mathrm{~g}$ of 4-oxazoline-2-thione (a) in $300 \mathrm{~mL}$ of benzene (thiophene free) or cyclohexane or a mixture of benzene and protic (methanol, ethanol or isopropanol) solvent was irmadiated with UV light from a Pyrex filtered $450 \mathrm{~W}$ Hanovia lamp for $10 \mathrm{~h}$ while oxygen was continuously bubbled into the solution. The course of the reaction was monitored by UV and TLC until the spot corresponding to the starting material disappeared.

Irradiation of 4,5-diphenyl-3-(4-methylphenyl)-4-oxazoline-2-thione (a) in benzene in presence of oxygen. A dilute solution containing $0.50 \mathrm{~g}$ of 4,5-diphenyl-3-(4-methylphenyl)4-oxazaline-2-thione in $300 \mathrm{~mL}$ of benzene was irradiated for $10 \mathrm{~h}$ according to the general procedure as described earlier. Three such runs were combined. The solvent was removed under reduced pressure and residue was triturated with ethanol to give $0.2 \mathrm{~g} \mathrm{(15 \% )} \mathrm{of} \mathrm{solid.}$ m.p. 142-144 ${ }^{\circ} \mathrm{C}$; identified as N,N-dibenzoyl-4-methylaniline. IR spectrum (Nujol, $\mathrm{cm}^{-1}$ ) $1700(\mathrm{VC}=0)$. NMR spectrum ( $\mathrm{CDCl}_{3}, \delta$ values): $7.4\left(\mathrm{~m}, 14 \mathrm{H}\right.$, arom); 2.3 (o, 3H. $\left.\mathrm{CH}_{3}\right)$. The mother liquor was concentrated and chromatographed on a column of neutral alumina ( $30 \mathrm{~g}$. $10 \times 2.2 \mathrm{~cm}$ ) and the results are given in Table 1 .

Table 1. Results of chromatographic analysis.

\begin{tabular}{|l|l|l|l|}
\hline Fractions $(50 \mathrm{~mL})$ & Eluent & Products & Yield \\
\hline $1-10$ & n-Hexane & - & - \\
\hline $11-18$ & n-Hexane & Solid & $0.096 \mathrm{~g}$ \\
\hline $19-30$ & n-Hexane-benzene $(1: 1)$ & Solid & $0.18 \mathrm{~g}$ \\
\hline $31-36$ & Benzene & Viscous liquid & Unidentified \\
\hline
\end{tabular}

Evaporation of solvent from the fractions 11-18 under reduced pressure afforded $0.096 \mathrm{~g}$ (21\%) of a solid, m:p. $95^{\circ} \mathrm{C}$, identified as benzil. The $\mathbb{R}$ spectrum was suprimposable to that of an authentic sample. Evaporation of solvent from the fractions 19-30 under reduced pressure gave $0.18 \mathrm{~g}(20 \%)$ of a solid identified as $\mathrm{N}-4$-methylphenyl benzamide, m.p. $157^{\circ} \mathrm{C}$, IR spectrum (Nujol, $\mathrm{cm}^{-1}$ ) $3300(\mathrm{VN}-\mathrm{H}), 1620(\mathrm{VC}=0)$ ). NMR spectrum (CDCl, 8 values): 7.50 (m, 9H, arom); 3.4 (bs, $\mathrm{H}, \mathrm{NH}, \mathrm{D}_{2} \mathrm{O}$ exchangeable); and 2.3 (s, 3H, $\mathrm{CH}_{3}$ ). Evaporation of the solvent from the fractions $31-36$ under reduced pressure gave a viscous liquid which. however, could not be identified.

Irradiation of 4,5-diphenyl-3-(4-methylphenyl)-4-oxazoline-2-thione (a) in cyclohexane in presence of oxygen. A dilute solution containing $0.50 \mathrm{~g}$ of 4,5-diphenyl-3-(4-methylphenyl). 4-oxazoline-2-thione in $300 \mathrm{~mL}$ of cyclohexane was irradiated for $10 \mathrm{~h}$ following the general procedure as described earlier. Three such runs were combined. The solvent was removed under reduced pressure and the residue was triturated with ethanol to give $0.2 \mathrm{~g}$ (15\%) of solid, identified as N,N-dibenzoyl-4-methylaniline as described earlier. The mother liquor was concentrated and chromatographed on a column of silica gel $(30 \mathrm{~g}, 12 \times 2.2 \mathrm{~cm})$ as deacribed earlier. Two products were obtained which were identified as benzil $(0.091 \mathrm{~g}, 20 \%)$ and $\mathrm{N}-4$ methylphenyl benzamide $(0.20 \mathrm{~g}, 22 \%)$ as described earlier when benzene is used as solvent. 
Methylene blue sensitized irradiation of 4,5-diphenyl-3-(4-methylphenyl)-4-oxazoline-2thione $(a)$ in benzene. A dilute solution containing $0.50 \mathrm{~g}$ of 4,5-diphenyl-3-(4methylphenyl)-4-oxazoline-2-thione in $300 \mathrm{~mL}$ of benzene and $0.05 \mathrm{~g}$ of methylene blue was taken in a quartz vessel and kept in sun light for $10 \mathrm{~h}$. Three such runs were combined. The unused methylene blue was removed by extraction with water. The solvent was removed under reduced pressure. The residue was triturated with $10 \mathrm{~mL}$ of ethanol to give $0.22 \mathrm{~g}$ (16\%) solid identified as N,N-dibenzoyl-4-methylaniline as described earlier. The mother liquor was concentrated and chromatographed on a column of silica gel $(30 \mathrm{~g}, 12 \times 2.2 \mathrm{~cm})$ as described earlier. Two products were obtained which were identified as benzil $0.082 \mathrm{~g}$, (18\%) and $\mathrm{N}$-4-methylphenyl benzamide $0.14 \mathrm{~g},(15.2 \%)$ as described earlier.

Irradiation of 4,5-diphenyl-3-(4-methylpheny()-4--oxazoline-2-thione (a) in benzene in nitrogen almosphere. A dilute solution containing $0.50 \mathrm{~g}$ of 4,5-diphenyl-3-(4-methylphenyl)4-oxazoline-2-thione in $300 \mathrm{~mL}$ of benzene (thiophene free) was irradiated with UV light from a Pyrex fittered 450W Hanovia lamp for $10 \mathrm{~h}$ while nitrogen was continuously bubbled into the solution to maintain nitrogen atmosphere and ensure proper mixing. Three such runs were combined. The solvent was removed under reduced pressure. The residue was chromatographed on a column of silica gel $(30 \mathrm{~g}, 12 \times 2.2 \mathrm{~cm})$ using petroleum ether as eluent. Chromatographic separation afforded two products identified as benzilmono-4methylphenylimine (0.89 g. 68.4\%) and 4,5-diphenyl-3-(4-methylphenyl)-4-0xazoline-2thione $(0)(0.45 \mathrm{~g}, 30 \%)$ on the basis of comparison (undepressed mixed melting point and superimposable IR spectrum) with authentic samples.

Irradiation of 4.5-diphenyl-3-(4-methylphenyl)-4-oxazoline-2-thione (a) in (1:1) methanolbenzene mixture in the presence of oxygen. A dilute solution containing $0.50 \mathrm{~g}$ of 4,5diphenyl-3-(4-methylphenyl)-4-oxazoline-2-thione in $300 \mathrm{~mL}$ of methanol-benzene mixture was irradiated for $10 \mathrm{~h}$ according to the general procedure as described earlier. Three such runs were combined. The solvent was removed under reduced pressure. The residue was triturated with ethanol to give $(0.13 \mathrm{~g} 9.4 \%)$ of a solid, m.p. $142-144^{\circ} \mathrm{C}$, identified as $\mathrm{N}, \mathrm{N}$ dibenzoly-4-methylaniline, IR spectrum (Nujol, $\left.\mathrm{cm}^{-1}\right) 1700$ (VC=0). NMR spectrum ( $\mathrm{CDCl}_{3}$, 8 values): $7.4\left(\mathrm{~m}, 14 \mathrm{H}\right.$, arom): $2.3\left(\mathrm{~s}, 3 \mathrm{H}, \mathrm{CH}_{3}\right)$. The mother liquor was concentrated and chromatographed on a column of silica gel $(30 \mathrm{~g}, 10 \times 2.2 \mathrm{~cm})$ and the results are given Table 2.

Table 2. Resules of chromatographic analysis.

\begin{tabular}{|l|l|l|l|}
\hline Practions $(50 \mathrm{~mL})$ & Eluent & Producis & Yield \\
\hline 1.10 & Pet.ether & - & - \\
\hline $11.1 \mathrm{~S}$ & Pet.ether & Solid & $0.054 \mathrm{~g}$ \\
\hline 16.20 & Pet.ether-benzene $(4: 1)$ & Solid & $0.074 \mathrm{~g}$ \\
\hline $21-30$ & Pet.ether-benzene $(1: 1)$ & Solid & $0.41 \mathrm{~g}$ \\
\hline 31.40 & Benzene & Viccous liquid & Unidentified \\
\hline
\end{tabular}

Evaporation of the solvent from the fractions $11-15$ afforded a solid $0.054 \mathrm{~g}$ (12\%), m.p. and mixed m.p. $95^{\circ} \mathrm{C}$. The IR spectrum was superimposable to that of an authentic sample of benzil. Evaporation of the solvent from the fractions 16-20 under reduced pressure gave a solid $0.074 \mathrm{~g}$ (14\%), m.p. and mixed m.p. $121^{\circ} \mathrm{C}$. The IR spectrum was suprimposable to that 
of an authentic sample of benzoic acid. Evaporation of the solvent from fractions 21-30 gave $0.41 \mathrm{~g}(45 \%)$ of a solid, m.p. $157^{\circ} \mathrm{C}$, identified as $\mathrm{N}-4$ methylphenyl benzamide, on the basis of spectral data as described earlier. Evaporation of the solvent from the fractions $31-40$ under reduced pressure gave a viscous liquid which, however, could not be identified.

Irradiation of 4,5-diphenyl-3-(4-methylphenyl)-4-oxazoline-2-thione (a) in ethanol-benzene (1:1) mixture in the presence of oxygen. A similar irradiation of a solution containing $0.50 \mathrm{~g}$ of -4-oxazoline-2-thione (a) in $300 \mathrm{~mL}$ of ethanol-benzene mixture according to the general procedure for $10 \mathrm{~h}$ and the usual work up of three such runs, as described earlier, gave four solid products which were identified as benzil $(0.059 \mathrm{~g}, 13 \%)$, benzoic acid $(0.069 \mathrm{~g}, 13 \%)$, $\mathrm{N}, \mathrm{N}$-dibenzoyl-4-methylaniline $(0.12 \mathrm{~g} 8.7 \%)$ and $\mathrm{N}-4$-methylphenyl benzamide $(0.38 \mathrm{~g}$, $41 \%)$.

Irradiation of 4,5-diphenyl-3-(4-methylphenyl)-4-oxazoline-2-thione (a) in 2-propanolbenzene (1:1) mixture in the presence of oxygen. A similar irradiation of a solution containing $0.50 \mathrm{~g}$ of -4-oxazoline-2-thione (a) in $300 \mathrm{~mL}$ of 2-propanol-benzene mixture according to the general procedure for $10 \mathrm{~h}$ and the usual work up of three such runs, as described earlier, gave four solid products which were identified as benzil $(0.045 \mathrm{~g}, 10 \%)$, benzoic acid $(0.085 \mathrm{~g}$. $16 \%), \mathrm{N}, \mathrm{N}$-dibenzoyl-4-methylaniline $(0.11 \mathrm{~g}, 8 \%)$ and $\mathrm{N}-4$-methylphenyl benzamide $(0.52$ $\mathrm{g}, 57 \%$ ) as described earlier.

Methylene blue sensitized irradiation of 4,5-diphenyl-3-(4-methylphenyl)-4-oxazoline-2thione $(a)$ in (1:1) benzene-methanol mixture in presence of oxygen. A dilute solution containing $0.50 \mathrm{~g}$ of 4,5-diphenyl-3-(4-methylphenyl)-4-oxazoline-2-thione in $300 \mathrm{~mL}$ of benzene and $0.05 \mathrm{~g}$ of methylene blue was taken in a quartz vessel and kept in sun light for 10 h. Three such runs were combined. The unused methylene blue was removed by extraction with water. The solvent was removed under reduced pressure. The residue was triturated with $10 \mathrm{~mL}$ of ethanol to give $(0.15 \mathrm{~g}, 11 \%)$ of solid identified as $\mathrm{N}, \mathrm{N}$-dibenzoyl-4-methylaniline as described earlier. The mother liquor was concentrated and chromatographed on a column of silica gel $(30 \mathrm{~g}, 12 \times 2.2 \mathrm{~cm})$ as described earlier. Three products were obtained which were identified as benzil $(0.045 \mathrm{~g}, 10 \%)$, N-4-methylphenyl benzamide $(0.39 \mathrm{~g}, 42 \%)$ and benzoic acid $(0.08 \mathrm{~g}, 15 \%)$ as described earlier.

Irradiation of 4,5-diphenyl-3-(4-methylphenyl)-4-oxazoline-2-thione(a) in $1: 1$ benzenemethanol mixture in nitrogen atmosphere. A dilute solution containing $0.50 \mathrm{~g}$ of (a) in 300 $\mathrm{mL}$ of benzene (thiophene free)- methanol mixture was irradiated with UV light from a Pyrex filtered 450W Hanovia lamp for $10 \mathrm{~h}$ while nitrogen was continuously bubbled into the solution to maintain nitrogen atmosphere and ensure proper mixing. Three such runs were combined. The solvent was removed under reduced pressure. The residue was chromatographed on a column of silica gel $(30 \mathrm{~g}, 12 \times 2.2 \mathrm{~cm})$ using petroleum ether as eluent. Chromatographic separation afforded two products identified as benzilmono-4methylphenylimine $(0.87 \mathrm{~g}, 67 \%)$ and 4,5-diphenyl-3-(4-methylphenyl)-4-oxazoline-2-thione (a), $(0.5 \mathrm{~g}, 33.3 \%)$ on the basis of comparison (undepressed mixed melting point and suprimposable $\mathbb{R}$ spectrum) with authentic samples. 


\section{CONCLUSION}

The photolysis of substituted -4-oxazoline-2-thione in aprotic solvents in oxygen atmosphere yields a 1,2-diketone, $\mathrm{N}$-substituted benzamide and $\mathrm{N}, \mathrm{N}$-diacyl $(\mathrm{RCO}$ or $\mathrm{Ar}-\mathrm{C}=\mathrm{O})$ amino derivative. The similar photolysis in protic solvent, in addition to above three products affords benzoic acid. The reaction route involves a dioxetane intermediate which cleaves into radicals to lead to the final products.

\section{ACKNOWLEDGEMENTS}

This work was sponsored by Copperbelt University. The author is thankful to the Head of the Department of Chemical Engineering, Mr. T. Kooma, for providing numerous facilities. Laboratory facilities from the Chemistry Department and useful discussion with Prof. K.N. Mehrotra at Banaras Hindu University (India) is gratefully acknowledged.

\section{REFERENCES}

1. Frump, J.A. Chem. Rev. 1971, 71, 483.

2. Reuman, M.; Meyers, A.I. Tetrahedron 1985, 41, 837.

3. Mehrotra, K.N.; Singh, I.S.; Roy, J. Bull. Chem. Soc. Jpn. 1985, 58, 2399.

4. Gomper, R.; Herlinger, H. Chem. Ber. 1956, 89, 2825.

5. Gagneux, A.R.; Goschke, R. Tetrahedron Lett. 1966, 5451.

6. Tsugea, O.; Oe, K.; Ueyama, Y. Chem. Letts. 1976, 425.

7. Stahlke, K.R.; Heins, H.G.; Hartman, W. Ann. Chem. 1972, 116, 764.

8. Vogel, A.I. Textbook of Practical Organic Chemistry, Sth ed., Longman: London;1989; p 916.

9. Padwa, A. J. Am. Chem. Soc 1965, 87, 4365 . 